\title{
Emission bands of nitrogen-implantation induced luminescent centers in ZnO crystals: Experiment and theory
}

\author{
X. M. Dai, ${ }^{1}$ S. J. Xu, ${ }^{1, a)}$ C. C. Ling, ${ }^{1}$ G. Brauer, ${ }^{2}$ W. Anwand, ${ }^{2}$ and W. Skorupa ${ }^{2}$ \\ ${ }^{1}$ Department of Physics, The University of Hong Kong, Pokfulam Road, Hong Kong, China \\ ${ }^{2}$ Institut für Ionenstrahlphysik und Materialforschung, Forschungszentrum Dresden-Rossendorf, \\ Postfach 510119, D-01314 Dresden, Germany
}

(Received 24 May 2012; accepted 9 August 2012; published online 30 August 2012)

\begin{abstract}
High quality $\mathrm{ZnO}$ crystals with the sharp band-edge excitonic emission and very weak green emission were implanted by nitrogen ions. An additional red emission band was observed in the asimplanted $\mathrm{ZnO}$ crystal and investigated as a function of temperature. By employing the underdamped multimode Brownian oscillator model for the general electron-phonon coupling system, both the original green and nitrogen-implantation induced red emission bands were theoretically reproduced at different temperatures. Excellent agreement between the theory and the experiment enables us determine the energetic positions of the pure electronic levels associated with the green and red emission bands, respectively. The determined energy level of the red emission band is in good agreement with the data obtained from the deep-level transient spectroscopy measurements. (C) 2012 American Institute of Physics.
\end{abstract}

[http://dx.doi.org/10.1063/1.4749402]

Zinc oxide $(\mathrm{ZnO})$ has been a technologically important material in a lot of fields due to its unique optical and electrical properties. Recently, it has attracted a renewed considerable interest due to its potential applications in photonics and optoelectronics. ${ }^{1}$ However, there are still some unsettled problems that stand in the way of realizing these uses. Among them are the difficulty of p-type doping and the insufficient understanding of the various defects. ${ }^{2,3}$ Asgrown $\mathrm{ZnO}$ always exhibits n-type electrical conductivity in the absence of intentional doping, and it is difficult to get reproducible and concentration-controllable p-type $\mathrm{ZnO}$. Nitrogen has been used as the p-type dopant in $\mathrm{ZnO}$ and some progress has been achieved along this direction. ${ }^{4}$ Defect-related broad emissions are commonly observed in the luminescence spectra of $\mathrm{N}$-doped $\mathrm{ZnO},{ }^{4}$ but the origin of the defects is far from full understanding. ${ }^{5}$ Getting a better understanding to these defects in $\mathrm{ZnO}$ is highly desirable. For nitrogen-implanted $\mathrm{ZnO}$, previous deep-level transient spectroscopy (DLTS) measurements show that there are two main deep levels located at $0.31 \mathrm{eV}$ (E3) and $0.95 \mathrm{eV}$ (D1) below the conduction band. ${ }^{6}$ The former is commonly found in as-grown $\mathrm{ZnO}$ single crystals, ${ }^{6,7}$ while the latter is confirmed to be created by the nitrogen implantation. ${ }^{6}$ On the other hand, deep centers in semiconductors and polar crystals are often optically active, which offers us the chance to investigate them with optical methods such as the photoluminescence (PL) technique.

In the present work, we attempt to obtain a better understanding of optically active deep centers in nitrogen-implanted $\mathrm{ZnO}$. In our study, a number of pre- and nitrogen-implanted $\mathrm{ZnO}$ samples were studied by the variable-temperature PL technique. In addition to the weak original green emission, a red emission band from the implanted samples was observed.

\footnotetext{
a) Author to whom correspondence should be addressed. Electronic mail: sjxu@hku.hk.
}

By employing the underdamped multimode Brownian oscillator (MBO) model recently developed for electron-phonon coupling systems, ${ }^{8,9}$ both the green and red emission bands in $\mathrm{ZnO}$ were theoretically reproduced. The pure electronic levels of the green and red emissions are, thus, determined and found to be consistent with the one of the DLTS detected levels $\left(\sim 0.9-1.0 \mathrm{eV}\right.$ below $\left.\mathrm{E}_{\mathrm{C}}\right)$ very well, which indicates that this $\mathrm{N}$-implantation induced deep centers are optically and electrically active.

The $\mathrm{ZnO}$ (0001) samples used in the experiments were commercially obtained from Cermet Inc., USA. The studied samples include one pre-implantation control sample and two implanted with different nitrogen ion doses: $10^{13} \mathrm{~cm}^{-2}$ and $10^{14} \mathrm{~cm}^{-2}$. During nitrogen implantation, the acceleration voltage of the nitrogen ion beam was $150 \mathrm{keV}$ while the sample temperature was set at $300{ }^{\circ} \mathrm{C}$. The implantation process and the $\mathrm{ZnO}$ samples used were identical to those employed in the previous DLTS study. ${ }^{6}$ Variable-temperature photoluminescence experiments were carried out on a PL system with a Janis closed cycle cryostat providing a temperature range from 4 to $300 \mathrm{~K}$. The $325 \mathrm{~nm}$ line of a Kimmon He-Cd continuouswave laser with an output power of $30 \mathrm{~mW}$ was employed as the excitation light source. Standard lock-in amplifier technology was also used to enhance the signal-to-noise ratio. Other details of the PL setup have been described elsewhere. ${ }^{10}$

Figure 1 shows the PL spectra in the visible spectral range measured from the pre-implantation sample at different temperatures. The inset illustrates the $4 \mathrm{~K}$ band-edge emissions of the sample in the ultraviolet (UV) spectral region. As reported in the literature, ${ }^{11}$ our pre-implantation sample emits dominant band-edge excitonic transitions and a very weak green band centered at about $500 \mathrm{~nm}$ when it was excited by the $325 \mathrm{~nm}$ UV laser, which indicates the high quality of the sample used in this study. Under the identical experimental conditions, PL spectra of the N-implanted samples were also measured at different temperatures. Unlike 


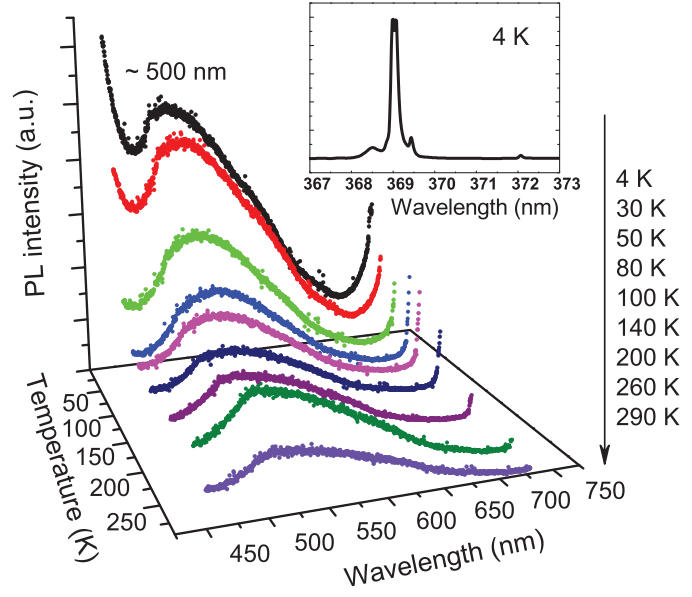

FIG. 1. PL spectra of the control sample at different temperatures. A weak green emission band centered at $\sim 500 \mathrm{~nm}$ can be found. The inset shows $4 \mathrm{~K}$ band-edge emissions of the sample.

the pre-implantation sample, the two implanted samples at low temperatures emit a noticeable red light band centered at $\sim 620 \mathrm{~nm}$ instead of the weak green emission, as shown in Fig. 2. Similar red emission band was also recently observed in $\mathrm{N}$-implanted $\mathrm{ZnO}$ bulk by Pan et al. ${ }^{12}$ As the temperature increases, the intensity of the red emission band goes down accompanied by a blueshift of the emission peak at higher temperatures above $200 \mathrm{~K}$. In the mean time, the relative intensity of the green luminescence band increases and becomes comparable to the red luminescence band at high temperatures. These phenomena indicate that the defect interactions may exist in the $\mathrm{N}$-implanted $\mathrm{ZnO}$ and lead to complex luminescence mechanisms of defects.

It is known that the color centers in wide gap polar crystals such as $\mathrm{ZnO}$ are usually treated as strong electron-phonon coupling systems. ${ }^{13}$ In the pioneer theoretical models, only

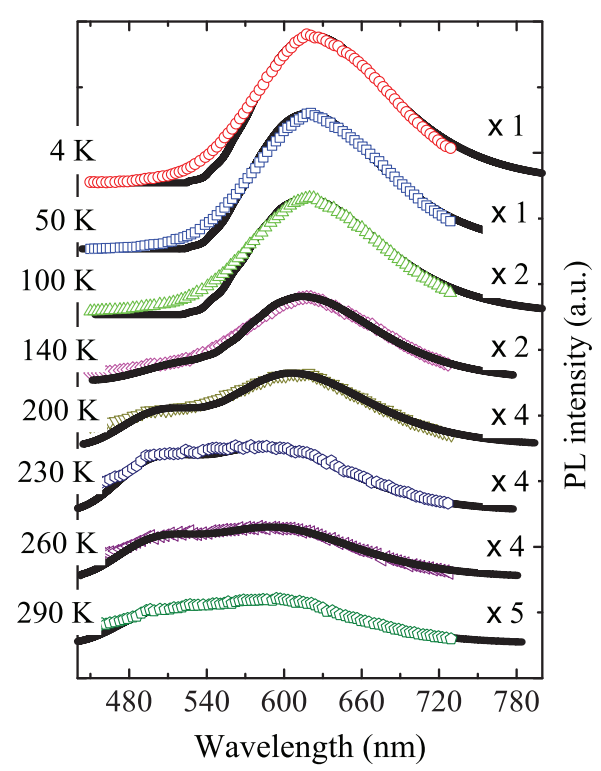

FIG. 2. Experimental and theoretical PL spectra at different temperatures for the $10^{13} \mathrm{~cm}^{-2} \mathrm{~N}$-implanted sample. The open symbols represent the experimental data while the solid lines are the theoretical spectra. Appropriate offset shifts in the vertical axis and the magnifications (times were marked) in the intensity were done to the curves for the simple clarity. coupling between electron and primary phonon such as longitudinal optical (LO) phonon was considered. ${ }^{14}$ Dissipation from an acoustical phonon bath was not taken into account. In the newly developed MBO model, ${ }^{8,9}$ some primary nuclear coordinates couple linearly to an electronic two-level system and simultaneously to a harmonic bath. Recently, the MBO model has been employed to reproduce PL spectra of InGaN quantum dots spontaneously formed in GaN matrix ${ }^{15}$ and the structured green emission band in $\mathrm{ZnO},{ }^{13}$ respectively. Here, we use the model to calculate both the red and green emission bands observed in the $\mathrm{N}$-implanted $\mathrm{ZnO}$. Following the previous study, ${ }^{13} 71.2 \mathrm{meV}$ or $574.2 \mathrm{~cm}^{-1}$ (the characteristic energy of the $\mathrm{LO}$ phonon in wurzite $\mathrm{ZnO}$ ) was adopted as the energy of the primary oscillator in our calculations. The frequency $\omega_{L O}$ of the primary oscillator, the damping coefficient $\gamma$ characterizing the coupling strength of the primary oscillator and the bath modes, and Huang-Rhys factor $S$ revealing the strength of the electron-LO phonon coupling, are the three key parameters of the MBO model.

In Fig. 2, the solid lines are the theoretical PL spectra of $10^{13} \mathrm{~cm}^{-2} \mathrm{~N}$-implanted sample at different temperatures by using the MBO model. For this sample, considering that the green emission band is relatively very weak at temperatures below $100 \mathrm{~K}$, we took it into account in the theoretical calculations only at temperatures higher than $100 \mathrm{~K}$. Such treatment leads to some discrepancy between theory and experiment at low temperatures for wavelengths below $\sim 575 \mathrm{~nm}$. From Fig. 2, it can be seen that a good agreement between the experiment and the theory is achieved in the whole interested temperature range. The parameter values used in a typical calculation (i.e., $\mathrm{T}=200 \mathrm{~K}$ ) were $\hbar \omega_{e g 1}^{o}=2.92 \mathrm{eV}, S_{1}=7.00$, $\gamma_{1}=65$, and $\hbar \omega_{e g 2}^{o}=2.38 \mathrm{eV}, S_{2}=5.25, \gamma_{2}=150$. The subscript 1(2) is for the green(red) emission. The weights for the green and red bands are 0.61 and 0.39 , respectively.

The first point that we would like to address in this section is the determination of the deep center levels from their broadening luminescence spectra due to the electron-phonon coupling. According to the electron-phonon coupling theory of color centers in wide gap crystals and semiconductors, ${ }^{14}$ strong electron-phonon coupling, i.e., Huang-Rhys factor $S \gg 1$, induces a red-shift $\left(\sim S \hbar \omega_{L O}\right)$ of the emission spectral center (peak) from the zero-phonon line (ZPL). In other words, only ZPL gives the real energetic position of the color center (defect) in these cases. From the ZPL values adopted in our calculations, the energetic positions of deep centers producing the green and red emission bands are determined to be 2.92 and $2.38 \mathrm{eV}$, respectively. Considering $3.30 \mathrm{eV}$ band gap of $\mathrm{ZnO}$ at room temperature, the defect levels of the green and red emission bands could be located at around $0.38 \mathrm{eV}$ and $0.92 \mathrm{eV}$ below the bottom of conduction band. The $0.92 \mathrm{eV}$ level coincides well with the D1 deep trap as detected in the previous DLTS study ${ }^{6}$ which involved the identical $\mathrm{N}$-implantation and $\mathrm{ZnO}$ samples. It is worthy to point out that the D1 deep traps were also observed in the Nimplanted $\mathrm{ZnO}$ sample after the identical annealing process at $300{ }^{\circ} \mathrm{C} .{ }^{6}$ It is thus plausible to conclude that the D1 trap is an intrinsic defect.

It is known that kinetic ions implanted into target material would collide with host atoms and subsequently push 
them away from their lattice sites. Therefore, the implantation always produces some intrinsic point defects such as vacancies and local lattice distortion in the targeted crystal. It is also known that oxygen vacancies have a low defect formation energy in the $\mathrm{ZnO}$ material, which has been demonstrated by first-principles calculations. ${ }^{16-19}$ Along with this line, the dominant electron deep levels (D1) in as-implanted $n-\mathrm{ZnO}$ could be tentatively assigned to the oxygen vacancy $V_{O}{ }^{6}$ The positron annihilation spectroscopy study carried out by Tuomisto et al. showed that the primary defect in the as-electron-irradiated $\mathrm{ZnO}$ (with electron energy of $2 \mathrm{MeV}$ ) was also identified as $V_{O}$ whose concentration is 10 times larger than that of $V_{Z n} \cdot{ }^{20}$ Furthermore, the $V_{O}$ was also identified to be the dominant donor-type defect in the $2.5 \mathrm{MeV}$ electron irradiated $\mathrm{ZnO}$ by using optical detection of electron paramagnetic resonance ${ }^{21}$ and in $\mathrm{P}^{+}$-implanted $\mathrm{ZnO}$ by Raman study. ${ }^{22}$ Vlasenko and Watkins suggested that the ZPL of the defect emission centered at $\sim 600 \mathrm{~nm}$ of the electron-irradiated $\mathrm{ZnO}$ was at $\sim 500 \mathrm{~nm}(2.48 \mathrm{eV}),{ }^{21}$ which was close to the ZPL of the red emission band in our Nimplanted sample. Before the electron-irradiation, their control $\mathrm{ZnO}$ samples emit a typical structureless green luminescence band when excited by an UV laser. Observations of the similar red emission bands in our $\mathrm{N}$-implanted $\mathrm{ZnO}$ and in Vlasenko et al. electron-irradiated samples indicate that one possible origin of the defects producing the red emission band is one kind of intrinsic defect, most likely oxygen vacancy. When nitrogen ions were injected into the $\mathrm{ZnO}$ crystal, they collide with the oxygen host atoms and make some oxygen atoms away from their equilibrium lattice sites. Such collision thus leads to generation of more oxygen vacancies. Furthermore, the charge particle implantation likely alters the charge state of most oxygen vacancies. According to Van de Walle's theoretical calculations, ${ }^{23,24}$ the appearing of a transition level E $(2+/ 0)$ offers the opportunity to emit a red light. The electron-LO phonon coupling and dissipation of continuous acoustic phonon bath also affect this luminescence process. Therefore, a broad red emission band is generated. From these available theoretical and experimental studies, the oxygen vacancy is the most possible microstructural origin causing the red emission band observed in $\mathrm{N}$-implanted $\mathrm{ZnO}$. As for the commonly observed green emission band without the periodic fine structures in $\mathrm{ZnO}$, the oxygen vacancy, but having the neutral charge state, is still the possible origin. ${ }^{25}$ From the electronic configurations of oxygen and zinc atoms as well as the unit crystalline structure of wurtzite $\mathrm{ZnO}$, an oxygen vacancy could have three different charge states: the neutral, $1+$, and $2+$, corresponding to the states of losing 0,1 , and 2 electrons, respectively. ${ }^{23}$ For the structured green emission band in $\mathrm{ZnO}$, copper impurities in $\mathrm{ZnO}$ are widely thought to be the resource because it is always observed in $\mathrm{Cu}$-doped $\mathrm{ZnO} .^{13,26,27}$ However, the defect characterization and firm identification in $\mathrm{ZnO}$ are still a challenging issue., ${ }^{2,3,5}$

A set of $\mathrm{ZnO}$ bulk crystals, including the as-grown and the ones implanted with different doses, have been studied in our present work. Two different colorful emissions, namely the common green band and the $\mathrm{N}$-implantation-caused red band, have been investigated both experimentally and theoretically. DLTS have detected two signals, namely D1 and E3 in the samples. The pure electronic level location of the implantation-induced red emission band was determined to be $0.92 \mathrm{eV}$ below the conduction band, which is in good agreement with the D1 center $(0.93 \mathrm{eV})$ detected by DLTS. Combining with the recent first-principles calculations and the other available experimental results, we suggest that the oxygen vacancy is the most possible origin of the N-implantation-caused red emission band in $\mathrm{ZnO}$.

This work was financially supported by the Hong Kong RGC-GRF Grants (Grant Nos. HKU705606P and 703612P), the Joint Research Fund for Overseas Chinese, Hong Kong and Macau Scientists of NSFC (Grant No. 61028012) and the HKU matching fund. One of the authors, SJX, would like to thank C. C. Zheng for his technical assistance in the replotting and format conversion of Fig. 2.

${ }^{1}$ Ü. Ozgür, Y. I. Alivov, C. Liu, A. Teke, M. A. Reshchikov, S. Doğan, V. Avrutin, S.-J. Cho, and H. Morkoç, J. Appl. Phys. 98, 041301 (2005).

${ }^{2}$ D. C. Look, Mater. Sci. Eng. B 80, 383 (2001).

${ }^{3}$ M. D. McCluskey and S. J. Jokela, J. Appl. Phys. 106, 071101 (2009).

${ }^{4}$ A. Tsukazaki, A. Ohtomo, T. Onuma, M. Ohtani, T. Makino, M. Sumiya,

K. Ohtani, S. F. Chichibu, S. Fuke, Y. Segawa, H. Ohno, H. Koinuma, and

M. Kawasaki, Nature Mater. 4, 42 (2005).

${ }^{5}$ A. Janotti and C. G. Van de Walle, Rep. Prog. Phys. 72, 126501 (2009).

${ }^{6}$ Q. L. Gu, C. C. Ling, G. Brauer, W. Anwand, W. Skorupa, Y. F. Hsu, A. B. Djurišić, C. Y. Zhu, S. Fung, and L. W. Lu, Appl. Phys. Lett. 92, 222109 (2008).

${ }^{7}$ J. C. Simpson and J. F. Cordaro, J. Appl. Phys. 63, 1781 (1988).

${ }^{8}$ S. Mukamel, Principles of Nonlinear Optical Spectroscopy (Oxford University Press, New York, 1995), p. 226.

${ }^{9}$ Y. Zhao and R. S. Knox, J. Phys. Chem. A 104, 7751 (2000).

${ }^{10}$ S. J. Xu, W. Liu, and M. F. Li, Appl. Phys. Lett. 77, 3376 (2000).

${ }^{11}$ D. W. Hamby, D. A. Lucca, and M. J. Klopfstein, J. Appl. Phys. 97, 043504 (2005).

${ }^{12}$ C. J. Pan, B. J. Pong, B. W. Chou, G. C. Chi, and C. W. Tu, Phys. Status Solidi C 3, 611 (2006).

${ }^{13}$ S. L. Shi, G. Q. Li, S. J. Xu, Y. Zhao, and G. H. Chen, J. Phys. Chem. B 110, 10475 (2006).

${ }^{14}$ K. Huang and A. Rhys, Proc. R. Soc. London, Ser. A 204, 406 (1950).

${ }^{15}$ S. J. Xu, G. Q. Li, Y. J. Wang, Y. Zhao, G. H. Chen, D. G. Zhao, H. Yang, D. P. Yu, and J. N. Wang, Appl. Phys. Lett. 88, 083123 (2006).

${ }^{16}$ A. F. Kohan, G. Ceder, D. Morgan, and C. G. Van de Walle, Phys. Rev. B 61, 15019 (2000).

${ }^{17}$ S. B. Zhang, S. H. Wei, and A. Zunger, Phys. Rev. B 63, 075205 (2001).

${ }^{18}$ J. L. Zhao, W. Q. Zhang, X. M. Li, G. W. Feng, and X. Shi, J. Phys.: Condens. Matter 18, 1495 (2006).

${ }^{19}$ F. Oba, S. R. Nishitani, S. Isotani, H. Adachi, and I. Tanaka, J. Appl. Phys. 90, 824 (2001).

${ }^{20}$ F. Tuomisto, K. Saarinen, D. C. Look, and G. C. Farlow, Phys. Rev. B 72, 085206 (2005).

${ }^{21}$ L. S. Vlasenko and G. D. Watkins, Phys. Rev. B 71, 125210 (2005).

${ }^{22}$ Z. Q. Chen, A. Kawasuso, Y. Xu, H. Naramoto, X. L. Yuan, T. Sekiguchi, R. Suzuki, and T. Ohdaira, J. Appl. Phys. 97, 13528 (2005).

${ }^{23}$ C. G. Van de Walle, Physica B 308, 899 (2001).

${ }^{24}$ A. Janotti and C. G. Van de Walle, Appl. Phys. Lett. 87, 122102 (2005).

${ }^{25}$ Y. W. Heo, D. P. Norton, and S. J. Pearton, J. Appl. Phys. 98, 0735023 (2005).

${ }^{26}$ R. Dingle, Phys. Rev. Lett. 23, 579 (1969).

${ }^{27}$ N. Y. Graces, L. Wang, L. Bai, N. C. Giles, L. E. Halliburton, and G. Cantwell, Appl. Phys. Lett. 81, 622 (2002). 